\title{
Essais
}

Revue interdisciplinaire d'Humanités

$2 \mid 2012$

Aux marges de l'humain

\section{Éric Dufour, Le Cinéma de science-fiction}

\section{Florent Favard}

\section{(2) OpenEdition}

Journals

Édition électronique

URL : https://journals.openedition.org/essais/10560

DOI : 10.4000/essais. 10560

ISSN : 2276-0970

\section{Éditeur}

École doctorale Montaigne Humanités

\section{Édition imprimée}

Date de publication : 15 novembre 2012

Pagination : 120-124

ISBN : 978-2-86781-857-8

ISSN : 2417-4211

\section{Référence électronique}

Florent Favard, «Éric Dufour, Le Cinéma de science-fiction », Essais [En ligne], 2 | 2012, mis en ligne le 17 janvier 2022, consulté le 20 janvier 2022. URL : http://journals.openedition.org/essais/10560 ; DOI : https://doi.org/10.4000/essais. 10560 
Lémergence de femmes dans le cinéma français a permis des récits centrés sur des personnages féminins qui ne sont pas étrangers à cette intimité brutale que Palmer tente de définir. L'auteur travaille sur des films familiaux comme LOL (L. Azuelos), Mes stars et moi (L. Colombani) et également sur des œuvres plus difficiles comme Et toi t'es sur qui? (L. Doillon) ou Innocence (L. Hadzihalilovic). Les violences faites aux femmes, le désir au féminin sont des sujets qui émergent de cette nouvelle tendance. Tim Palmer fait la comparaison avec les États-Unis qui ont attendu 2010 pour donner l'Oscar du meilleur réalisateur à une femme, Kathryn Bigelow, pour Démineurs.

Pour conclure Tim Palmer présente en détail la FEMIS. L'école envisage la formation de ses cinéastes comme un temps de recherche artistique. Les promotions majoritairement féminines dans les années 1990 ont modifié le paysage cinématographique français. Tim Palmer surestime l'importance de la FEMIS car de nombreux réalisateurs ont réussi sans passer entre ses murs. La cinéphilie, même si elle a beaucoup évolué, reste pour lui le support sur lequel s'est bâti le cinéma français contemporain.

Louvrage de Tim Palmer propose une belle synthèse sur le cinéma français des années 2000: un cinéma brutal qui touche à l'intime grâce à une forme basée sur la frontalité et la violence. L'émergence de la figure féminine comme personnage et comme réalisatrice est l'évolution majeure constatée par l'auteur. Sa connaissance de la culture française et de ses institutions n'empêche pas Tim Palmer de porter un regard distancié sur un cinéma qu'il aime passionnément. Il éclaire sous un jour nouveau le cinéma français à travers des problématiques basées sur les statistiques du CNC, la réception des films Art et Essai aux ÉtatsUnis et les relations des institutions culturelles françaises avec le jeune cinéma. Malgré des chapitres maladroitement agencés, Brutal Intimacy constitue un support de qualité pour l'étude du cinéma français contemporain.

Laurie Deson-Leiner, MICA EA 4426, lauriedeson@yahoo.fr

\section{Éric DUFOUR, Le Cinéma de science-fiction, Paris, Armand Colin, 2011}

Avec Le Cinéma de science-fiction, paru en 2011, le philosophe Éric Dufour poursuit son analyse des films de genre après, notamment, Le Cinéma d'horreur et ses figures publié aux Presses Universitaires de France en 2006. Dans son dernier ouvrage, il prend pour objet un autre "mauvais genre, un genre méprisé ", constatant par-là un intérêt récent pour les films de S-F, principalement dans la recherche anglo-saxonne. Évitant les approches strictement historique ou thématique, Dufour livre une analyse systématique en se basant sur un corpus très étendu, qui part de la préhistoire du cinéma à nos jours en transcendant les frontières - quand bien même son principal intérêt réside dans la cinématographie américaine, qu'il considère comme fondatrice 
du cinéma de science-fiction. L'ouvrage alterne deux perspectives : la première, diachronique, se pose comme une histoire de la création et de l'évolution du genre jusqu'aux années 1970, à partir desquelles Dufour passe à un point de vue synchronique, analysant successivement l'esthétique puis la politique du film de S-F contemporain.

Partant de Méliès et de son film comique Le Voyage dans la Lune (1902), qui "semble être le premier film de S-F", Dufour commence par passer en revue tout le cinéma d'avant la Seconde Guerre mondiale: il s'arrête ainsi pêle-mêle sur le Paris qui dort de René Clair (1927), la maquette monumentale érigée pour Metropolis (Fritz Lang, 1927) ou encore les serials, " films découpés en épisodes ", mettant en scène le héros Flash Gordon, issu des comic strips. Il insiste sur le fait que ces ouvres ne peuvent se reposer que sur l'héritage de la littérature de science-fiction, le genre n'ayant encore aucune légitimité au cinéma, notamment aux États-Unis où, s'il prospère, il reste affilié au genre de l'horreur. Le cinéma de science-fiction est encore dans sa préhistoire.

C'est là, l'originalité de la perspective diachronique de l'auteur: conscient qu'un genre ne naît qu'en se reposant sur des bases établies, en "prenant le visage de l'ancien et [...] en dévoilant progressivement sa physionomie inédite ", Dufour place la naissance du genre au cinéma dans les années 1950, aux États-Unis. Il la lie à la perte de pouvoir des grands studios, qui laissent le champ libre aux minors et surtout aux indépendants. À eux seuls, ces derniers vont "faire " le cinéma de science-fiction, attirant dans des drive-in flambant neufs un nouveau public constitué d'adolescents. Pour la première fois, les films s'affirment comme appartenant au genre S-F. Riche de possibilités mais n'ayant pas encore construit son identité, le cinéma de science-fiction va s'inspirer d'autres genres, ce qui permet à l'auteur de distinguer la S-F scientifique, rigoureuse, la S-F film de monstre, la S-F film noir ou encore la S-F space opera, héritière de Flash Gordon et consorts. Cette typologie vise à cerner les codes qui vont progressivement émerger dans le genre S-F: la transgression de la règle des $180^{\circ}$ issue de l'horreur, l'image de la Terre vue de l'espace avant même les premiers clichés d'Explorer 6 en 1959, jusqu'à la volonté des bandes-annonces de mettre en avant le concept d'une œuvre. Dufour souligne aussi la fonction performative du langage, "qui consiste à créer des événements qui n'existent pas en dehors de lui ", et qui prend racine dans ce jeune cinéma de S-F. Elle permet d'escamoter des explications scientifiques et laisse souvent hors-champ une technologie extraordinaire, pour réduire, par exemple, un passage en hyperespace à sa simple mention par le commandant de bord du vaisseau. L'auteur s'attarde d'ailleurs sur la transition supposée entre nomination et monstration dans les années 1980, spécifiant que « la S-F est fondée sur le goût d'inventer des mots » et qu'aujourd'hui encore 
perdurent au cinéma des explications scientifiques évoquant des technologies fabuleuses, que l'image peine à remplacer.

Dufour voit les années 1960 comme le pivot du genre S-F au cinéma: son succès aux États-Unis lui fait traverser les frontières, et pendant un temps apparaissent des S-F nationales. Il s'attarde sur le Japon et le fantôme des bombardements de Hiroshima et Nagasaki, présent dès 1954 dans le Gojira de Ishirō Honda; le cinéma britannique, dans lequel se développe le sous-genre S-F film politique à l'aune des adaptations de 1984, de Georges Orwell; la $\mathrm{S}$-F italienne, mêlant comédie, effets-spéciaux et érotisme; enfin le courant français, et les films des jeunes turcs, de La Jetée de Chris Marker (1962) à l'Alphaville de Godard (1965), qui mettent en exergue la capacité du cinéma de science-fiction à influer non seulement sur le fond d'une ouvre, mais aussi sur sa forme. Cette dernière caractéristique, esthétique, se retrouve ensuite dans tout film de $\mathrm{S}-\mathrm{F}$ " qui prétend faire plus que simplement raconter une histoire ", par exemple avec Solaris (1972) et Stalker (1979) d'Andrei Tarkovsky. C'est d'ailleurs l'exemple du cinéma de S-F soviétique, de qualité, pacifiste et prosélyte, que Dufour emploie pour expliquer, via sa cannibalisation par le cinéma américain, le brouillage des frontières à l'aube des années 1970. Partant des remontages "sauvages " opérés sur les œuvres originales, purgées de leur message communiste et mâtinées de scènes additionnelles tournées aux ÉtatsUnis, l'auteur élargit la problématique et constate la lente uniformisation d'un cinéma de science-fiction qui transcende les frontières. Les échanges entre les cultures occidentale (notamment américaine) et japonaise illustrent ce phénomène: le mouvement cyberpunk, pleinement reconnu à partir des années 1980, est issu de la convergence de topoi S-F des deux côtés du Pacifique.

Arguant que les spécificités nationales des cinémas de science-fiction se noient dans une uniformisation mondiale, Dufour passe ainsi d'une perspective diachronique à une autre, synchronique, plus proche de son précédent ouvrage sur le genre de l'horreur. Il y trace une cartographie des imaginaires du cinéma de science-fiction contemporain, explorant des critères esthétiques et politiques. On pourrait contester le point de vue de l'auteur sur la disparition des caractéristiques nationales des films de S-F, à même de maintenir une typologie influencée par des cultures variées. Il n'en reste pas moins que la seconde partie de l'ouvrage est la plus intéressante, car Dufour peut y exprimer pleinement son point de vue de philosophe sur un genre cinématographique peu étudié au sein de la recherche française.

Son esthétique du cinéma de S-F, loin d'une approche thématique, dévoile le fonctionnement du récit audiovisuel de science-fiction, commençant par l'évidence, "l'imaginaire visuel du voyage dans l'espace ", la révolution numérique et les expériences formelles sur l'image, pour ensuite s'attarder sur la notion fondamentale de sublime, rappelant que Kant distinguait le sublime 
mathématique (en S-F, le changement d'unité de grandeur, l'impossibilité de mesurer les objets dans le plan) et le sublime dynamique (le déchaînement de la nature menaçant le héros, pleinement servi par les prouesses numériques). De la désorientation propre au genre à l'anthropomorphisme dans la représentation de l'extraterrestre, de l'Autre, jusqu'à la mise en scène du posthumain et de la machine, et des questions qu' elle soulève, Dufour dresse le panorama d'une esthétique riche de possibles et trop souvent ravalée au rang de spectacle gratuit, en évitant un effet catalogue par la constante élaboration de liens entre ces topoi.

Plus aventureuse, plus inédite peut-être aussi, est sa politique du film de S-F, où Dufour s'interroge longuement sur la capacité du genre à fonctionner comme une critique sociale, notant que c'est là sa principale destination. Au travers de ses questionnements sur le devenir de l'humanité et de notre société, le cinéma de $\mathrm{S}-\mathrm{F}$ nous renvoie sans cesse à notre monde, monde duquel il est issu et ne peut se séparer, limitant peut-être sa capacité à penser "out of the box". Après avoir interrogé la place de Dieu dans le genre, l'auteur se lance dans une ambitieuse typologie des dystopies, des films d'anticipation nous prévenant des dérives certaines d'un futur proche, aux œuvres qui osent "faire surgir les impensés de notre propre société ", comme par exemple la remise en cause des normes sexuées. Dufour reste prudent, se posant toujours la question du point de vue adopté par ces dénonciations, et de ce qu'elles disent, notamment, de la manipulation des images et des opinions. Un discours qu'il développe en évoquant l'omniprésence, dans le cinéma de science-fiction, des sociétés totalitaristes sous toutes leurs formes. Jouant le jeu des différences entre l'adaptation de 1984 par Michael Radford (1984), Brazil (Terry Gilliam, 1985) ou encore THX 1138 (George Lucas, 1972), Dufour note que la conception du totalitarisme a changé depuis la Seconde Guerre mondiale, passant du communisme tout puissant, du Parti de l'œuvre d'Orwell, aux sociétés ultra-libérales contrôlant les individus "sans parti ni idéologie apparents ». Un totalitarisme qui, donc, ne se donne pas comme tel, amenant Dufour à emprunter à Denis Vernant l'idée de totalitarismisation, système dans lequel l'individu se retrouve « instrumentalisé par un dispositif à la mise en place duquel il a lui-même participé ». Ce changement du paradigme totalitaire dans le cinéma de science-fiction, Dufour le rattache, dans l'ultime chapitre de l'ouvrage, à une analyse pertinente de l'évolution du sous-genre cyberpunk, mêlant codes du film noir, informatique et transgression de l'ordre social, et « où le politique s'est dissous dans l'économique ».

Éric Dufour apporte avec cet ouvrage un éclairage nouveau sur un genre cinématographique encore peu abordé par la recherche française. Si ses prises de positions peuvent en dérouter certains - notamment une perspective diachronique qui sort des sentiers battus, et une constante comparaison avec 
l'esthétique des jeux vidéos, certes justifiée mais souvent peu subtile -, Le Cinéma de science-fiction offre une approche résolument nouvelle en France sur les films de S-F, et parvient à tirer, dans sa seconde partie, l'essence même du genre. Il propose en outre une réflexion poussée sur la critique sociale de la S-F postmoderniste, suffisamment complète pour permettre à l'ouvrage d'anticiper sur le développement futur du genre.

Florent Favard, MICA EA 4426, favard.florent@gmail.com 